Daguang Wang, Wei Li and Yabin Sun*

\title{
Crystal structure of poly $\left[\left(\mu_{2}-9 \mathrm{H}\right.\right.$-carbazole-3,6-dicarboxylate-

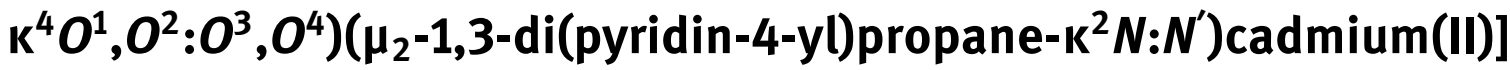 monohydrate, $\mathrm{C}_{27} \mathrm{H}_{23} \mathrm{~N}_{3} \mathrm{O}_{5} \mathrm{Cd}$
}

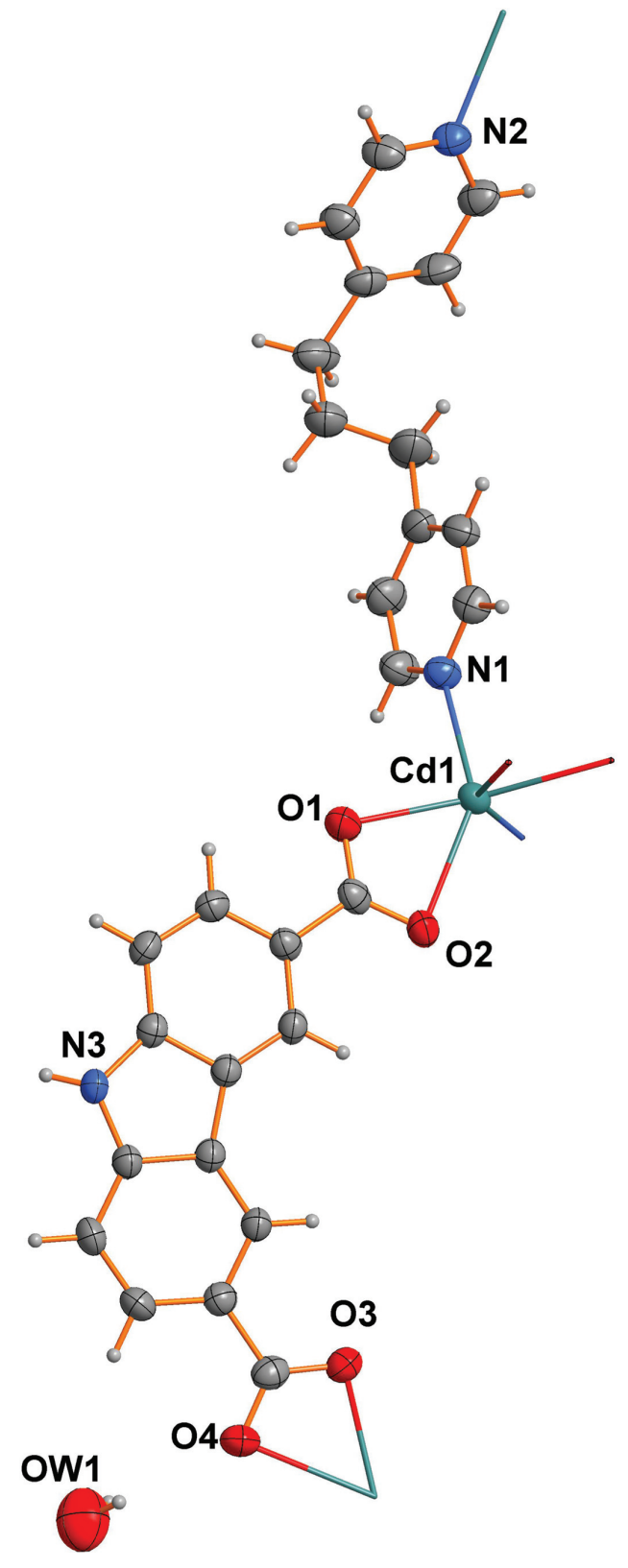

*Corresponding author: Yabin Sun, Department of Ophthalmology, The First Hospital of Jilin University, Changchun 130021, P.R. China, e-mail: drsunyb@protonmail.com

Daguang Wang and Wei Li: Department of Gastric and Colorectal Surgery, The First Hospital of Jilin University, Changchun, Jilin, 130021, China https://doi.org/10.1515/ncrs-2019-0307

Received April 29, 2019; accepted June 20, 2019; available

online August 29, 2019

\author{
Abstract \\ $\mathrm{C}_{27} \mathrm{H}_{23} \mathrm{~N}_{3} \mathrm{O}_{5} \mathrm{Cd}$, monoclinic, $P 2_{1} / n$ (no. 14), $a=9.6161(7) \AA$, \\ $b=16.5427(11) \AA, \quad c=15.8900(11) \AA, \quad \beta=105.653(1)^{\circ}$, \\ $V=2434.0(3) \AA^{3}, Z=4, R_{\mathrm{gt}}(F)=0.0327, w R_{\mathrm{ref}}\left(F^{2}\right)=0.0828$, \\ $T=152(2) \mathrm{K}$.
}

CCDC no.: 1935425

A part of the molecular structure is shown in the figure. Table 1 contains crystallographic data and Table 2 contains the list of the atoms including atomic coordinates and displacement parameters.

Table 1: Data collection and handling.

\begin{tabular}{ll}
\hline Crystal: & Colourless block \\
Size: & $0.26 \times 0.25 \times 0.22 \mathrm{~mm}$ \\
Wavelength: & Mo $K \alpha$ radiation $(0.71073 \AA$ A $)$ \\
$\mu:$ & $0.94 \mathrm{~mm}^{-1}$ \\
Diffractometer, scan mode: & Apex $2, \varphi$ and $\omega$ \\
$\theta_{\text {max }}$, completeness: & $28.3^{\circ},>99 \%$ \\
$N(h k l)_{\text {measured }}, N(h k l)_{\text {unique }}, R_{\text {int }}:$ & $15410,5948,0.027$ \\
Criterion for $I_{\text {obs }}, N\left(h k l_{\text {gt }}:\right.$ & $I_{\text {obs }}>2 \sigma\left(I_{\text {obs }}\right), 4588$ \\
$N(\text { param })_{\text {refined }}:$ & 331 \\
Programs: & Bruker [1], SHELX [2, 3] \\
\hline
\end{tabular}

\section{Source of material}

A mixture of $9 H$-carbazole-3,6-dicarboxylic acid $\left(\mathrm{H}_{2} \mathrm{~L}\right)$ (0.2 mmol), 4-(3-(pyridin-4-yl)propyl)pyridine $(0.2 \mathrm{mmol})$, $\mathrm{CdCl}_{2} \cdot 2.5\left(\mathrm{H}_{2} \mathrm{O}\right)(0.2 \mathrm{mmol})$, and $\mathrm{H}_{2} \mathrm{O}(20 \mathrm{~mL})$ was stirred for ten minutes. The mixture was transferred in a $25 \mathrm{~mL}$ stainless steel reactor with a Teflon liner and heated from 298 to $453 \mathrm{~K}$ in $5 \mathrm{~h}$ and a constant temperature was maintained at $453 \mathrm{~K}$ for $72 \mathrm{~h}$. After cooling to room temperature, pink block crystals were collected in $58.7 \%$ yield based on $\mathrm{Cd}$.

\section{Experimental details}

The $U_{\text {iso }}$ values of the hydrogen atoms of parts of carbon group were set to $1.2 U_{\mathrm{eq}}(\mathrm{C})$. Hydrogen atoms of water molecules were located from electron density map. 
Table 2: Fractional atomic coordinates and isotropic or equivalent isotropic displacement parameters $\left(\AA^{2}\right)$.

\begin{tabular}{|c|c|c|c|c|}
\hline Atom & $x$ & $y$ & $z$ & $U_{\text {iso }} * / U_{\text {eq }}$ \\
\hline $\mathrm{C} 1$ & $0.7966(3)$ & $0.19533(17)$ & $0.84541(16)$ & $0.0356(6)$ \\
\hline $\mathrm{C} 2$ & $0.7232(3)$ & $0.21621(16)$ & $0.91397(15)$ & $0.0322(5)$ \\
\hline C3 & $0.7654(3)$ & $0.17707(16)$ & $0.99529(16)$ & $0.0353(6)$ \\
\hline $\mathrm{H} 3 \mathrm{~A}$ & 0.8367 & 0.1359 & 1.0042 & $0.042^{\star}$ \\
\hline C4 & $0.7064(3)$ & $0.19660(16)$ & $1.06251(16)$ & $0.0363(6)$ \\
\hline H4 & 0.7342 & 0.1688 & 1.1168 & $0.044^{\star}$ \\
\hline C5 & $0.6051(3)$ & $0.25817(15)$ & $1.04818(16)$ & $0.0321(5)$ \\
\hline C6 & $0.5566(3)$ & $0.29717(15)$ & $0.96594(15)$ & $0.0313(5)$ \\
\hline C7 & $0.6174(3)$ & $0.27528(16)$ & $0.89904(15)$ & $0.0335(6)$ \\
\hline H7 & 0.5865 & 0.3007 & 0.8435 & $0.040^{*}$ \\
\hline $\mathrm{C} 8$ & $0.4555(3)$ & $0.35887(15)$ & $0.97601(15)$ & $0.0316(5)$ \\
\hline C9 & $0.4500(3)$ & $0.35468(15)$ & $1.06382(16)$ & $0.0332(5)$ \\
\hline C10 & $0.3666(3)$ & $0.40879(17)$ & $1.09639(17)$ & $0.0411(6)$ \\
\hline $\mathrm{H} 10$ & 0.3627 & 0.4057 & 1.1554 & $0.049^{\star}$ \\
\hline C11 & $0.2899(3)$ & $0.46693(17)$ & $1.04088(17)$ & $0.0412(6)$ \\
\hline H11 & 0.2330 & 0.5045 & 1.0623 & $0.049^{\star}$ \\
\hline C12 & $0.2937(3)$ & $0.47196(16)$ & $0.95339(16)$ & $0.0345(6)$ \\
\hline C13 & $0.3767(3)$ & $0.41797(15)$ & $0.92107(16)$ & $0.0336(6)$ \\
\hline H13 & 0.3799 & 0.4213 & 0.8619 & $0.040^{*}$ \\
\hline C14 & $0.2094(3)$ & $0.53496(16)$ & $0.89399(18)$ & $0.0382(6)$ \\
\hline C15 & $1.1725(3)$ & $0.00172(17)$ & $0.74655(18)$ & $0.0400(6)$ \\
\hline H15 & 1.0923 & -0.0266 & 0.7106 & $0.048^{*}$ \\
\hline C16 & $1.3061(3)$ & $-0.03501(18)$ & $0.76672(18)$ & $0.0453(7)$ \\
\hline H16 & 1.3161 & -0.0878 & 0.7455 & $0.054^{\star}$ \\
\hline C17 & $1.4255(3)$ & $0.00490(19)$ & $0.81776(18)$ & $0.0446(7)$ \\
\hline C18 & $1.4014(3)$ & $0.0803(2)$ & $0.84946(19)$ & $0.0500(7)$ \\
\hline H18 & 1.4793 & 0.1094 & 0.8867 & $0.060^{\star}$ \\
\hline C19 & $1.2642(3)$ & $0.11322(19)$ & $0.82678(19)$ & $0.0460(7)$ \\
\hline H19 & 1.2504 & 0.1651 & 0.8489 & $0.055^{\star}$ \\
\hline $\mathrm{C} 20$ & $1.5780(4)$ & $-0.0280(2)$ & $0.8365(2)$ & $0.0670(10)$ \\
\hline $\mathrm{H} 20 \mathrm{~A}$ & 1.5762 & -0.0783 & 0.8025 & $0.080^{*}$ \\
\hline $\mathrm{H} 20 \mathrm{~B}$ & 1.6386 & 0.0117 & 0.8160 & $0.080^{*}$ \\
\hline C21 & $1.6462(3)$ & $-0.0457(2)$ & $0.9305(2)$ & $0.0550(8)$ \\
\hline $\mathrm{H} 21 \mathrm{~A}$ & 1.6032 & -0.0956 & 0.9471 & $0.066^{\star}$ \\
\hline $\mathrm{H} 21 \mathrm{~B}$ & 1.6251 & -0.0008 & 0.9665 & $0.066^{*}$ \\
\hline C22 & $1.8097(3)$ & $-0.0565(2)$ & $0.9504(2)$ & $0.0545(8)$ \\
\hline $\mathrm{H} 22 \mathrm{~A}$ & 1.8524 & -0.0058 & 0.9354 & $0.065^{\star}$ \\
\hline $\mathrm{H} 22 \mathrm{~B}$ & 1.8495 & -0.0656 & 1.0140 & $0.065^{\star}$ \\
\hline C23 & $1.8552(3)$ & $-0.12518(17)$ & $0.90210(19)$ & $0.0413(7)$ \\
\hline C24 & $1.9012(3)$ & $-0.11294(19)$ & $0.8285(2)$ & $0.0519(8)$ \\
\hline $\mathrm{H} 24$ & 1.8994 & -0.0600 & 0.8050 & $0.062^{\star}$ \\
\hline C25 & $1.9500(3)$ & $-0.17694(19)$ & $0.7883(2)$ & $0.0469(7)$ \\
\hline H25 & 1.9812 & -0.1668 & 0.7374 & $0.056^{*}$ \\
\hline C26 & $1.9054(3)$ & $-0.26502(18)$ & $0.8883(2)$ & $0.0462(7)$ \\
\hline H26 & 1.9045 & -0.3187 & 0.9094 & $0.055^{\star}$ \\
\hline C27 & $1.8555(3)$ & $-0.20385(18)$ & $0.93116(19)$ & $0.0466(7)$ \\
\hline $\mathrm{H} 27$ & 1.8213 & -0.2157 & 0.9806 & $0.056^{\star}$ \\
\hline N1 & $1.1504(2)$ & $0.07537(14)$ & $0.77522(14)$ & $0.0384(5)$ \\
\hline N2 & $1.9549(2)$ & $-0.25264(14)$ & $0.81869(15)$ & $0.0386(5)$ \\
\hline N3 & $0.5363(2)$ & $0.29235(13)$ & $1.10510(13)$ & $0.0359(5)$ \\
\hline
\end{tabular}

Table 2 (continued)

\begin{tabular}{lrrrr}
\hline Atom & $\boldsymbol{x}$ & $\boldsymbol{y}$ & $\boldsymbol{z}$ & $\boldsymbol{U}_{\text {iso }}{ }^{*} \boldsymbol{U}_{\text {eq }}$ \\
\hline H3 & 0.5461 & 0.2767 & 1.1593 & $0.043^{*}$ \\
O1 & $0.9035(2)$ & $0.14850(13)$ & $0.86485(13)$ & $0.0518(5)$ \\
O2 & $0.75157(19)$ & $0.22488(12)$ & $0.76958(11)$ & $0.0425(5)$ \\
O3 & $0.2180(2)$ & $0.53754(12)$ & $0.81603(12)$ & $0.0494(5)$ \\
O4 & $0.1318(2)$ & $0.58407(13)$ & $0.92068(13)$ & $0.0552(6)$ \\
OW1 & $0.0378(4)$ & $0.6251(2)$ & $1.0814(2)$ & $0.0981(10)$ \\
Cd1 & $0.93072(2)$ & $0.14287(2)$ & $0.72699(2)$ & $0.03374(7)$ \\
H1A & $0.113(2)$ & $0.6379(17)$ & $1.0609(19)$ & $0.051^{*}$ \\
H2A & $-0.001(3)$ & $0.5898(16)$ & $1.0401(17)$ & $0.051^{*}$ \\
\hline
\end{tabular}

\section{Comment}

The research of coordination polymers (CPs) is significant and unparalleled for their diversified, designable and tailorable structures as well as unique chemical and physical properties. Over the past decades, CPs have been paid much attention for their great potential applications in many areas [5-8]. Coordination polymers are constructed by incorporating metal cations, anionic and/or neutral linkers. In order to obtain a targeted coordination polymer, it is essential to select appropriate linkers. Polycarboxylates represent one type of anionic linkers for the construction of coordination polymers.

In the title complex, there is one crystallographically independent cadmium(II) ions, one dicarboxylato ligand (L), one 4-(3-(pyridin-4-yl)propyl)pyridine and one water molecule. The cadmium(II) is six-coordinated by four $\mathrm{O}$ atoms from two L ligands, and two $\mathrm{N}$ atoms from two 4-(3-(pyridin4-yl)propyl)pyridine ligands to form a distorted octahedron. The distances between $\mathrm{Cd}$ and the coordinated atoms are: $\mathrm{d}(\mathrm{Cd} 1-\mathrm{N} 1)=2.330(2) \AA, \mathrm{d}(\mathrm{Cd} 1-\mathrm{N} 2)=2.271(2) \AA, \mathrm{d}(\mathrm{Cd} 1-\mathrm{O} 1)=$ 2.2777(18) $\AA, \quad \mathrm{d}(\mathrm{Cd} 1-\mathrm{O} 2)=2.4270(17) \AA, \quad \mathrm{d}(\mathrm{Cd} 1-03)=$ 2.2393(18) $\AA$, and $d(C d 1-04)=2.4581(19) \AA$, respectively. The $\mathrm{Cd}(\mathrm{II})$ coordination angles are in the normal range from $55.22(6)^{\circ}$ to $150.69(7)^{\circ}$. The $\mathrm{Cd}(\mathrm{II})$ ions are bridged by L ligands and 4-(3-(pyridin-4-yl)propyl)pyridine ligands into a 1D-network. The similar 1D structures have been reported, for example, $\left[\mathrm{Cu}(\right.$ bipa $\left.)(\mathrm{py})_{2}\right] \cdot 0.5\left(\mathrm{H}_{2} \mathrm{O}\right)$ prepared from the solvothermal reaction of 5-bromoisophthalic acid, $\mathrm{Cu}\left(\mathrm{NO}_{3}\right)_{2} \cdot 3 \mathrm{H}_{2} \mathrm{O}$ and pyridine, and [Co(p-cpdba)(2,2'bpy) $]_{n}$ prepared from 4-(4-carboxyphenylamino)-3,5dinitrobenzolate acid, $\mathrm{CoCl}_{2} \cdot 6 \mathrm{H}_{2} \mathrm{O}$ and 2,2'-bipyridine. Both are 1D chain structures through cocoordinating interactions between metal ions with oxygen atoms and nitrogen atoms $[9,10]$. In addition, nitrogen ligands are mostly used as the second ligand to obtain more diverse structres. There are many directly related structures with 4-(3-(pyridin-4-yl) propyl)pyridine as nitrogen ligand, such as [11-14]. 
Acknowledgements: This work is supported by the project development plan of science and technology of Jilin Province.

\section{References}

1. Bruker. APEX2, SAINT and SADABS. Bruker AXS Inc., Madison, WI, USA (2009).

2. Sheldrick, G. M.: SHELXT - integrated space-group and crystal-structure determination. Acta Crystallogr. A71 (2015) 3-8.

3. Sheldrick, G. M.: Crystal structure refinement with SHELXL. Acta Crystallogr. C71 (2015) 3-8.

4. Huang, X.; Zhang, S.; Liu, L.; Yu, L.; Chen, G.; Xu, W.; Zhu, D.: Superconductivity in a copper(II)-based coordination polymer with perfect kagome structure. Angew. Chem. Int. Ed. 57 (2018) 146-150.

5. Shang, K.-X.; Sun, J.; Hu, D.-C.; Yao, X.-Q.; Zhi, L.-H.; Si, C.-D.; Liu, J.-C.: Six Ln (III) coordination polymers with a semirigid tetracarboxylic acid ligand: bifunctional luminescence sensing, NIR-luminescent emission, and magnetic properties. Cryst. Growth Des. 18 (2018) 2112-2120.

6. Yang, Y.; Wang, K.-Z.; Yan, D.: Smart luminescent coordination polymers toward multimode logic gates: time-resolved, tribochromic and excitation-dependent fluorescence/ phosphorescence emission. ACS Appl. Mater. Interfaces 9 (2017) 17399-17407.

7. Yang, Y.; Zhu, W.; Dong, Z.; Chao, Y.; Xu, L.; Chen, M.; Liu, Z.: 1D Coordination polymer nanofibers for low-temperature photothermal therapy. Adv. Mater. 29 (2017) 1703588.

8. Zhang, R.; Gong, Q.; Emge, T. J.; Banerjee, D.; Li, J.: Design and synthesis of new $1 \mathrm{D}$ and $2 \mathrm{R}$-isophthalic acid-based coordination polymers $(R=$ hydrogen or bromine). Chimia 67 (2013) 393-396.

9. Feng, G.; Xu, H.; Li, W.; Zhang, J.: Resolution of chiral nitrogen atoms in $1 \mathrm{D}$ helical coordination polymers. Inorg. Chem. Commun. 96 (2018) 81-85.

10. Zhang, X.; Bing, Y.; Gao, P.; Bai, H.; Hu, M.: High sensitive luminescent sensing for nitrobenzene and iron(III) by uncommon Ln-MOFs containing open ketone group sites. Inorg. Chim. Acta 455 (2017) 98-104.

11. Yan, M.-J.; Feng, Q.; Song, H.-H.: Synthesis and photoluminescence properties of silver(I) complexes based on $\mathrm{N}$-benzoylL-glutamic acid and $\mathrm{N}$-donor ligands with different flexibility. J. Mol. Struct. 1112 (2016) 147-154.

12. Guo, X.; Guo, H.; Zou, H.; Qi, Y.; Liu, C.: Tuning the entanglement of coordination polymers by controlling the steric hindrance of organic linkers. J. Mol. Struct. 1043 (2013) 116-120.

13. Gui-Lian, L.; Jia, S.; Yu-Ying, H.: Hydrothermal synthesis and crystal structure of poly[aqua-( $\mu_{2}-1,3$-bis(4-pyridyl) propane- $\left.\mathrm{K}^{2} N: N^{\prime}\right)-\left(\mu_{2}-1,4,5,6,7,7-\right.$ hexachlorobicyclo[2.2.1] hept-5-ene-2,3-dicarboxylato- $\mathrm{K}^{2} \mathrm{O}: \mathrm{O}^{\prime}$ )manganese(II) hydrate, $\mathrm{C}_{22} \mathrm{H}_{20} \mathrm{Cl}_{6} \mathrm{~N}_{2} \mathrm{O}_{6} \mathrm{Mn}$. Z. Kristallogr. NCS 233 (2018) 95-97.

14. Ling-Yun, X.; Zhou-Ru, J.; Rong-Rong, G.: Crystal structure of diaqua-bis(3,5-dichloroisonicotinato- ${ }^{1} O$ )-bis(1,3-di(pyridin-4yl)propane- ${ }^{1} N$ )cobalt(II), $\mathrm{C}_{38} \mathrm{H}_{36} \mathrm{Cl}_{4} \mathrm{~N}_{6} \mathrm{O}_{6} \mathrm{Co}$. Z. Kristallogr. NCS 234 (2019) 495-497. 